\title{
ERRATUM: “THE PROPER MOTION OF THE GALACTIC CENTER PULSAR RELATIVE TO SAGITTARIUS A*” $(2015$, ApJ, 798, 120)
}

\author{
Geoffrey C. Bower ${ }^{1}$, Adam Deller ${ }^{2}$, Paul Demorest ${ }^{3}$, Andreas Brunthaler ${ }^{4}$, Heino Falcke $^{2,4,5}$, \\ Monika Moscibrodzka ${ }^{5}$, Ryan M. O'Leary ${ }^{6}$, Ralph P. Eatough ${ }^{4}$, Michael Kramer ${ }^{4,7}$, K. J. Lee ${ }^{4}$, Laura Spitler ${ }^{4}$, \\ Gregory Desvignes ${ }^{4}$, Anthony P. Rushton ${ }^{8,9}$, Sheperd Doeleman ${ }^{10,11}$, and Mark J. Reid ${ }^{11}$ \\ ${ }^{1}$ Academia Sinica Institute of Astronomy and Astrophysics, 645 N. A'ohoku Place, Hilo, HI 96720, USA; gbower@asiaa.sinica.edu.tw \\ ${ }^{2}$ ASTRON, P.O. Box 2, 7990 AA Dwingeloo, The Netherlands \\ ${ }^{3}$ NRAO, 520 Edgemont Road, Charlottesville, VA 22903-2475, USA \\ ${ }^{4}$ Max-Planck-Institut für Radioastronomie, Auf dem Hügel 69, D-53121 Bonn, Germany
${ }^{5}$ Department of Astrophysics, Institute for Mathematics, Astrophysics and Particle Physics (IMAPP), Radboud University, \\ PO Box 9010, 6500 GL Nijmegen, The Netherlands \\ ${ }^{6}$ JILA, University of Colorado and NIST, 440 UCB, Boulder, CO 80309-0440, USA \\ ${ }^{7}$ Jodrell Bank Centre of Astrophysics, University of Manchester, Manchester M13 9PL, UK \\ ${ }^{8}$ Department of Physics, Astrophysics, University of Oxford, Keble Road, Oxford OX1 3RH, UK \\ ${ }^{9}$ School of Physics and Astronomy, University of Southampton, Highfield, Southampton SO17 1BJ, UK \\ ${ }^{10}$ MIT Haystack Observatory, Route 40, Westford, MA 01886, USA \\ ${ }^{11}$ Harvard-Smithsonian Center for Astrophysics, 60 Garden Street, Cambridge, MA 02138, USA \\ Received 2016 March 11; accepted 2016 March 12; published 2016 April 22
}

We used an incorrect value for the ICRF position of Sgr $\mathrm{A}^{*}$ at the specified reference date to determine the relative position of the magnetar, PSR J1745-2900, in our analysis. The correct value for the Sgr A* position is $17 \mathrm{~h} 45 \mathrm{~m} 40.0366 \mathrm{~s},-29 \mathrm{~d} 00^{\prime} 28^{\prime \prime} 217$ at epoch $\mathrm{MJD}=56710$. ICRF positions reported for the magnetar are correct as reported in the paper, but relative positions should be adjusted by $(+18.36,-32.0)$ mas. This applies to individual epoch positions reported in columns 6 and 7 of Table 2 and the mean fitted positions reported in rows 1 and 2 of Table 4. For example, the correct fitted offset position using the proper motion bootstrap technique in Table 4 is $+1700.53,-1679.41$ mas. The relative positions in Figure 1 should be shifted by the same offset. Proper motion, acceleration, and core shift values are unaffected. There are no changes in the interpretation of the results.

Table 2

Observed Positions of PSR J1745-2900

\begin{tabular}{ccccccc}
\hline \hline MJD & Band & $\begin{array}{c}\text { Flux Density } \\
(\mathrm{mJy})\end{array}$ & $\begin{array}{c}\text { ICRF RA } \\
(\text { J2000 })\end{array}$ & $\begin{array}{c}\text { ICRF Dec. } \\
(\text { J2000 })\end{array}$ & $\begin{array}{c}\Delta \alpha \\
(\mathrm{mas})\end{array}$ \\
\hline 56422 & $\mathrm{X}$ & 0.56 & $174540.166377 \pm 0.000020$ & $-290029.8960 \pm 00.0002$ & $1699.97 \pm 0.26$ \\
56444 & $\mathrm{X}$ & 0.76 & $174540.166327 \pm 0.000012$ & $-290029.8962 \pm 00.0001$ & $1699.50 \pm 0.16$ \\
56473 & $\mathrm{Ku}$ & 0.58 & $174540.166263 \pm 0.000008$ & $-290029.8962 \pm 00.0001$ & $1698.91 \pm 0.10$ \\
56486 & $\mathrm{X}$ & 1.47 & $174540.166222 \pm 0.000008$ & $-290029.8965 \pm 00.0001$ & $1698.49 \pm 0.10$ & $-1683.24 \pm 0.1682 .80 \pm 0.10$ \\
56658 & $\mathrm{Ku}$ & 2.09 & $174540.166264 \pm 0.000007$ & $-290029.8960 \pm 00.0001$ & $1700.52 \pm 0.09$ & $-1679.79 \pm 0.10$ \\
$\ldots$ & $\mathrm{X}$ & 1.18 & $174540.166276 \pm 0.000010$ & $-290029.8960 \pm 00.0001$ & $1700.68 \pm 0.13$ & $-1679.79 \pm 0.10$ \\
56710 & $\mathrm{Ku}$ & 1.07 & $174540.166249 \pm 0.000005$ & $-290029.8960 \pm 00.0001$ & $1700.77 \pm 0.07$ & $-1679.00 \pm 0.10$ \\
$\ldots$ & $\mathrm{X}$ & 0.94 & $174540.166237 \pm 0.000014$ & $-290029.8962 \pm 00.0002$ & $1700.62 \pm 0.18$ & $-1679.20 \pm 0.20$ \\
56750 & $\mathrm{~K}$ & 0.92 & $174540.166235 \pm 0.0000004$ & $-290029.8961 \pm 00.0001$ & $1700.94 \pm 0.05$ & $-1678.49 \pm 0.10$ \\
$\ldots$ & $\mathrm{Q}$ & 0.54 & $174540.166238 \pm 0.0000003$ & $-290029.8959 \pm 00.0001$ & $1700.98 \pm 0.04$ & $-1678.29 \pm 0.10$ \\
56772 & $\mathrm{X}$ & 1.00 & $174540.166246 \pm 0.000016$ & $-290029.8958 \pm 00.0002$ & $1701.27 \pm 0.21$ & $-1677.86 \pm 0.20$ \\
$\ldots$ & $\mathrm{Ku}$ & 1.22 & $174540.166204 \pm 0.0000008$ & $-290029.8961 \pm 00.0001$ & $1700.72 \pm 0.10$ & $-1678.16 \pm 0.10$ \\
56892 & $\mathrm{Ku}$ & 0.63 & $174540.166196 \pm 0.000028$ & $-290029.8959 \pm 00.0003$ & $1701.65 \pm 0.37$ & $-1676.15 \pm 0.30$ \\
56899 & $\mathrm{~K}$ & 0.26 & $174540.166250 \pm 0.000025$ & $-290029.8959 \pm 00.0003$ & $1702.42 \pm 0.33$ & $-1676.06 \pm 0.30$ \\
$\ldots$ & $\mathrm{Q}$ & 0.15 & $174540.166215 \pm 0.000005$ & $-290029.8958 \pm 00.0001$ & $1701.95 \pm 0.07$ & $-1675.90 \pm 0.10$ \\
\hline & & & & &
\end{tabular}

Table 4

Proper Motion Fits for PSR J1745-2900

\begin{tabular}{|c|c|c|c|c|c|}
\hline Parameter & Units & PM Bootstrap & PM LSQ & PM + Accel. & PM + Core Shift \\
\hline$\overline{\Delta \alpha_{0}}$ & (mas) & $1700.53 \pm 0.07$ & $1700.52 \pm 0.07$ & $1700.54 \pm 0.09$ & $1700.52 \pm 0.19$ \\
\hline$\Delta \delta_{0}$ & (mas) & $-1679.41 \pm 0.03$ & $-1679.41 \pm 0.03$ & $-1679.42 \pm 0.05$ & $-1679.35 \pm 0.10$ \\
\hline$\mu_{\alpha}$ & (mas/yr) & $2.45 \pm 0.33$ & $2.50 \pm 0.22$ & $2.49 \pm 0.23$ & $2.51 \pm 0.31$ \\
\hline$\mu_{\delta}$ & (mas/yr) & $5.88 \pm 0.11$ & $5.90 \pm 0.09$ & $5.92 \pm 0.10$ & $5.84 \pm 0.12$ \\
\hline$a_{\alpha}$ & $\left(\mathrm{mas} / \mathrm{yr}^{2}\right)$ & $\ldots$ & $\ldots$ & $-0.2 \pm 1.0$ & $\ldots$ \\
\hline$a_{\delta}$ & $\left(\mathrm{mas} / \mathrm{yr}^{2}\right)$ & $\ldots$ & $\ldots$ & $0.2 \pm 0.5$ & $\ldots$ \\
\hline$\Phi_{\alpha}$ & $(\mathrm{mas} / \mathrm{cm})$ & $\ldots$ & $\ldots$ & $\ldots$ & $-0.00 \pm 0.10$ \\
\hline$\Phi_{\delta}$ & $(\mathrm{mas} / \mathrm{cm})$ & $\ldots$ & $\ldots$ & $\ldots$ & $0.03 \pm 0.05$ \\
\hline$\chi_{\alpha}^{2} / d . o . f_{\cdot \alpha}$ & $\ldots$ & $\ldots$ & $112.3 / 13$ & $111.8 / 12$ & $112.3 / 12$ \\
\hline$\chi_{\delta}^{2} / d . o . f \cdot \delta$ & $\ldots$ & $\ldots$ & $17.0 / 13$ & $16.8 / 12$ & $16.5 / 12$ \\
\hline
\end{tabular}



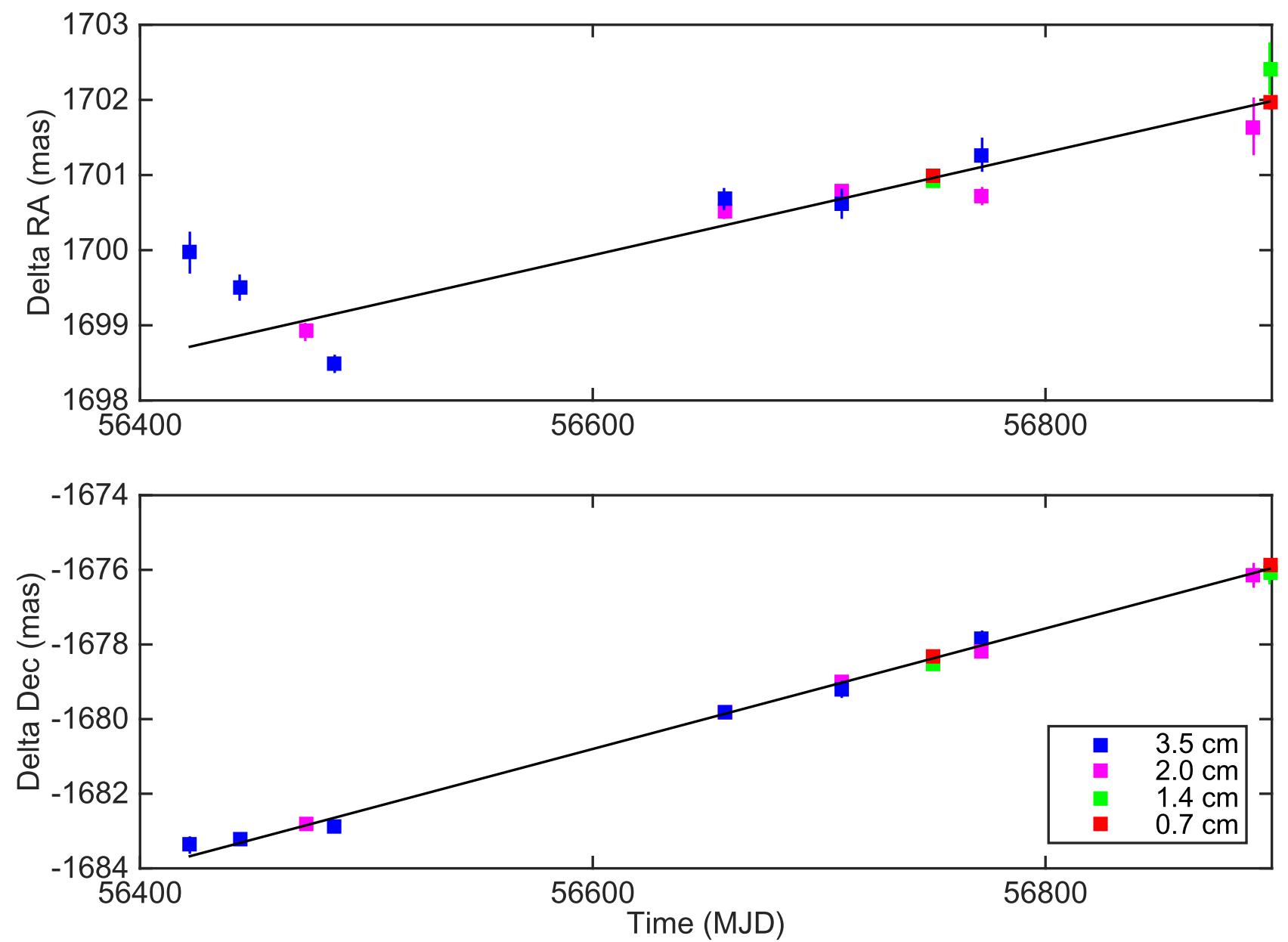

Figure 1. Position as a function of time for PSR J1745-2900 relative to Sgr A*. Different colors are used to identify the wavelength of observations. The solid black line shows the best-fit proper motion in each coordinate (see, text for details of the fit). 